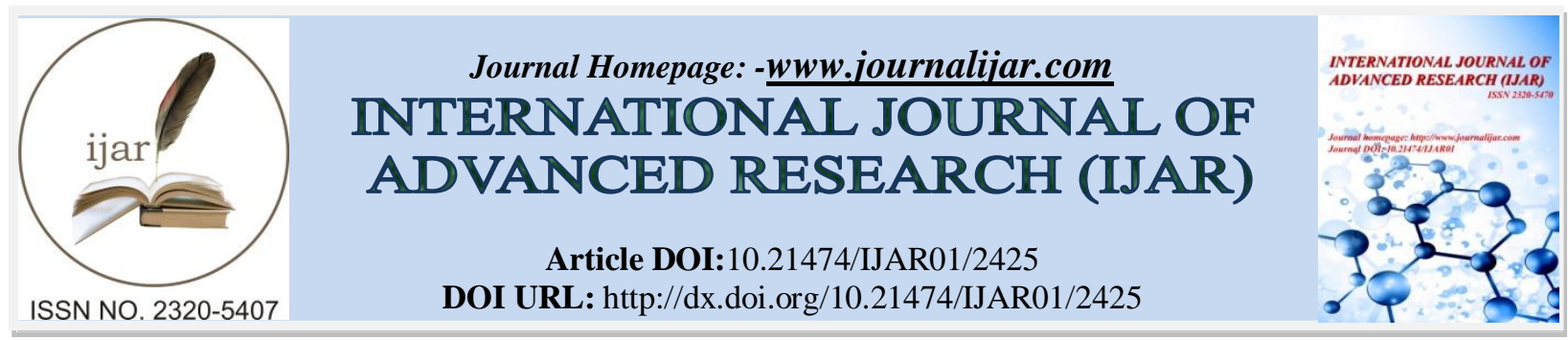

RESEARCH ARTICLE

\title{
ASSESSMENT OF PHONOPHORESIS OF CORTICOSTEROIDS IN THE TREATMENT OF MILD TO MODERATE CARPAL TUNNEL SYNDROME.
}

Mohammad A. Zakaria.

Physical Medicine, Rheumatology and Rehabilitation Department, Faculty of Medicine, Ain Shams University, Cairo, Egypt.

\section{Manuscript Info}

Manuscript History

Received: 20 October 2016

Final Accepted: 22 November 2016

Published: December 2016

Key words:

Carpal tunnel syndrome, Phonophoresis, local corticosteroid injection.

\section{Abstract}

Background: Carpal tunnel syndrome (CTS) is the most commonly diagnosed disabling condition of the upper extremities. Local steroid injections (LSI) are widely used for management of CTS. Phonophoresis is popular method in transdermal medication delivery that may eliminate potential side effects which can occur by LSI.

Objectives: To investigate the efficacy of phonophoresis of corticosteroids in the treatment of mild to moderate CTS and to compare it with LSI.

Patients and methods: Forty patients with mild to moderate CTS (56 hands) were included in this study and randomly assigned into 2 groups. One group received phonophoresis of $0.4 \%$ dexamethasone sodium phosphate (Dex-P) using continuous ultrasound $1 \mathrm{MHz}, 5-\mathrm{cm}^{2}$ probe, $1.0 \mathrm{~W} / \mathrm{cm}^{2}, 10 \mathrm{~min} / \mathrm{session}, 3$ sessions/week for 4 weeks. The other group received LSI of $0.4 \mathrm{mg}$ Dex-P using the classic proximal approach. Outcome measures were performed before and one month after treatment and included pain assessment by visual analogue scale, symptoms severity scale and the functional status scale of the Boston Carpal Tunnel Questionnaire, hand grip strength and electrodiagnostic studies (motor and sensory distal latencies of the median nerve).

Results: There was a statistically significant improvement in all outcome measures in both groups one month after treatment compared with baseline $(\mathrm{P}<0.05)$. No statistically significant difference was found between the two groups in any outcome measure one month after treatment $(\mathrm{P}>0.05)$.

Conclusion:Phonophoresis of corticosteroids could be an effective treatment in mild to moderate CTS and may be an alternative treatment to LSI.

Copy Right, IJAR, 2016. All rights reserved.

\section{Introduction}

Carpal tunnel syndrome (CTS) is the most common entrapment mononeuropathy. It arises from entrapment (mechanical compression) of the median nerve at the wrist. It accounts for $90 \%$ of all entrapment neuropathies and it is particularly prevalent in middle-aged women and the dominant hand is commonly affected [1,2]. Prolonged mechanical compression of the nerve can result in ischemia and demyelination of the nerve and in severe cases 
axonal loss [3]. CTS is characterized by numbness, tingling and/or burning sensation in the sensory distribution of the median nerve in the hand, which may be accompanied by pain and/or weakness in the affected hand. CTS can result in limitation of activities of daily living, loss of sleep and work disability [2,4]. The delay in diagnosis and treatment of CTS may result in irreversible median nerve damage with persistent symptoms and permanent disability [4,5]. CTS is basically diagnosed by symptoms, signs and electro-diagnostic studies. Patients often have Tinel's sign, Phalen's sign, reverse Phalen's sign, flick sign, and positive Durkan's test. The electro-diagnosis for CTS requires neurophysiological evidence of impaired median nerve conduction tests across the wrist [6].Various possibilities of treatment including surgical and conservative treatments have been used for CTS. Conservative treatments include rest \& avoidance of extreme activity with the hand, splinting of the wristin a neutral position, physical therapy modalities like ultrasound \& laser therapy, non-steroidal anti-inflammatory drugs (NSAIDs) and local corticosteroid injections [4].

Local steroid injections (LSI) are widely used in the management of CTS; however, it can cause complications such as ischemia, skin depigmentation and atrophy and trauma to superficial flexor muscle tendons of the hand. Median nerve injury is the most serious complication associated with local steroid injection for CTS [7].

Phonophoresis is a technique by which therapeutic ultrasound (US) is used to introduce pharmacological agents, usually anti-inflammatory drugs, through intact skin into the inflamed subcutaneus tissues. Its major advantages are the introduction of medication to a local area without invasion of the skin and the synergistic interaction of US and drugs. Phonophoresis can provide a safe and painless alternative to injections for treatment of many inflammatory musculoskeletal disorders such as CTS, bursitis and tendinitis [8,9]. Corticosteroids are widely used with phonophoresis in physical therapy because they possess marked anti-inflammatory effect and are available in relatively cheap forms designed for topical administration. [10].

Several tools have been used to assess the outcome of CTS treatment. The nerve conduction studies (NCS) are the most important ones. Median motor \& sensory distal latencies (MDL \& SDL) are widely accepted as sensitive parameters for measuring changes in CTS [10-13].

\section{Aim of the work}

This study was designed to evaluate the efficacy of phonophoresis of corticosteroids in the treatment of patients with mild to moderate CTS and to compare the results to those obtained with LSI, a treatment known to be effective in mild to moderate CTS.

\section{Patients and methods}

Patients were recruited from the outpatient clinic of the Physical medicine, Rheumatology and Rehabilitation department, Faculty of Medicine, Ain Shams University and the study was performed in the department.The study was approved by the research ethics committee of the Faculty of Medicine, Ain Shams University, and all procedures were in accordance with the Helsinki Declarations of 1975. Written informed consents were obtained from all patients after explanation of the purpose and procedures of the study.

All patients were diagnosed as clinical CTS as per the criteria of The American Academy of Neurology practice parameters [14-15] as follows:

1. Paresthesia, pain, swelling, weakness, or clumsiness of the hand provoked or worsened by sleep, sustained hand or arm position, or repetitive action of the hand or wrist that is decreased by changing posture or by hand shaking.

2. Sensory deficits in the median nerve innervated region of the hand.

3. Motor deficit or hypotrophy of the median nerve innervated thenar muscles.

4. Positive provocative clinical tests (Phalen's maneuver and/or Tinel's sign).

Clinical CTS was defined if criterion 1 and one or more of criteria 2-4 were fulfilled.

Electro-diagnostic studies (EDX) of median and ulnar nerves of the both hands were carried out for all patients by the same physician as per the recommended protocol of the American Association of Neuromuscular \&Electrodiagnostic Medicine (AANEM) [12] using Schwarzertopas basic EMG system version 1.401, a division of Natus Europe $\mathrm{GmbH}$, Germany. All EDX studies were performed in a room temperature $25^{\circ} \mathrm{C}$ and the skin temperature of the forearm and the wrist was kept at $32^{\circ} \mathrm{C}$ during all measurements. The EDX cut-off points (normal values) we used in our study were the following $[10,15,16]$ : 
1. Median nerve distal sensory latency (DSL) measured to peak $\leq 3.5 \mathrm{~ms}$.

2. Median nerve distal motor latency $(\mathrm{DML}) \leq 4.2 \mathrm{~ms}$.

3. Difference between the median \& ulnar nerve DSLs of the same hand $\leq 0.4 \mathrm{~ms}$.

4. Difference between the median \& ulnar nerve DMLs of the same hand $\leq 1 \mathrm{~ms}$.

Patients were categorized into 6 groups of severity as per a scale given by Bland [17] as follows:

1. Very mild (grade 1): CTS demonstrable only with most sensitive tests (e.g. inching).

2. Mild (grade 2): abnormal DSL, normal DML.

3. Moderate (grade 3): abnormal DSL, abnormal DML but $<6.5 \mathrm{~ms}$.

4. Severe (grade 4): absence of sensory response, abnormal DML but $<6.5 \mathrm{~ms}$.

5. Very severe (grade 5): absence of sensory response, abnormal DML $>6.5 \mathrm{~ms}$.

6. Extremely severe (grade 6): sensory and motor potentials effectively unrecordable (surface motor potential from APB $<0.2 \mathrm{mV}$ amplitude).

Only patients with mild to moderate CTS (grades $2 \& 3$ ) with symptoms lasting more than one month were included in this study.

Patients were excluded if they had severe or extreme CTS, thenar eminence hypotrophy or atrophy, associated ulnar neuropathy, diabetes or systemic peripheral neuropathies. Patients were also excluded if they had history of thyroid disease, wrist arthritis or history of steroid injection into the carpal tunnel, previously treated with ultrasound, lowlevel laser therapy or iontophoresis for CTS.

Forty patients, 8 males and 32 females were included in this study; 16 patients with both hands aff ected (14 females $\& 2$ males) and 24 patients with the right hand aff ected (18 females \& 6 males). All patients included in the study were right handed. Patients (males \& females); with (both wrists \& single wrist affected) were randomly assigned into one of two groups: Group A, received phonophoresis intervention and Group B, received LSI.

All patients were subjected to the following measurements before treatment and one months after the end of therapy.

1. Pain assessment by the patients using visual analogue scale (VAS), a 10-cm line ranging from 0 (no pain at all) to 10 (the most severe pain that I can imagine). A difference of $1.3 \mathrm{~cm}$ on a VAS represents the minimum change in acute pain that is clinically significant [18].

2. Assessment of the symptoms severity using the symptoms severity scale (SSS) and the functional impairment using the functional status scale (FSS). Both are parts of the Boston Carpal Tunnel Questionnaire (BCTQ). The SSS has 11 questions, the FSS has 8 questions, each question has a five-point scale ranges from 1 to 5 . Each scale generates a final score (sum of individual item scores divided by number of items), which ranges from 1 to 5. Higher SSS and FSS scores correlate with more severe symptoms and functional impairment, respectively[19]. This evaluation instrument was recognized asreproducible, valid, withinternal consistency andable to respond to clinicalchanges[20].

3. Hand grip strength was measured in Kilogram unit using a hand-held dynamometer (Camry Digital Hand Dynamometer, Model: EH101, Camry Electronic Co. Ltd, California, USA). The patient held the dynamometer in the hand to be tested while sitting with his or her shoulder adducted and neutrally rotated, elbow flexed at $90^{\circ}$, forearm and wrist neutrally positioned. Patients were instructed to squeeze the dynamometer as hard as they could with maximum effort for 5 seconds. No other body movement was allowed. This procedure was repeated 3 times with an interval of 30 seconds between trials. The mean of 3 trials' scores was recorded.

4. EDX.

\section{Treatment}

Group A: Received Phonophoresis of 0.4\% dexamethasone sodium phosphate (Dex-P) using EnrafNoniusSonopuls 590, Netherlands. Aquasonic gel containing 0.4\% Dex-P was applied over the area of the carpal tunnel. Continuous ultrasound waves with intensity of $1.0 \mathrm{~W} / \mathrm{cm}^{2}$ at a $1 \mathrm{MHz}$ frequency using the $5-\mathrm{cm}^{2}$ probe was utilized to transfer the medication.All patients received 12 treatment sessions of phonophoresis, $10 \mathrm{~min} / \mathrm{session}, 3$ days/week, for 4 weeks. During this period and for 1 months after the end of the therapy, patients were not allowed to use any other form of therapy, splinting or drugs.

Group B: Received LSI in the carpal tunnel. An injection of $4 \mathrm{mg}$ Dex-P ( $1 \mathrm{ml}$ of Fortecortin $8 \mathrm{mg} / 2 \mathrm{ml}$; Merk, Germany) using a 25 -gauge needle. All injections were performed by the same physician. The patient was in seated 
position, with forearm in supination and wrist slightly dorsiflexed. The palmaris longus tendon was determined by resistive wrist flexion, the injection was made just medial to the tendon. The needle was inserted $2 \mathrm{~cm}$ proximal to the first (most distal) wrist crease at an angle of 45 degrees toward the palm. If the palmaris longus tendon was absent, the injection was carried out just medial to the midline of the ventral aspect of the wrist. If paresthesia was elicited, the needle was withdrawn and redirected more medially. Patients were advised to actively move their fingers for several minutes to allow distribution of the solution. Patients were reviewed one month after the injection. During this period, patients were not allowed to use any other form of therapy, splinting or drugs.

\section{Statistical Analysis}

IBM SPSS statistics (V. 24.0, 2016; IBM Corp., Chicago, Illinois, USA) was used for data analysis. Data were expressed as Mean \pm SD for quantitative parametric measures in addition to both number and percentage for categorized data. Comparison between two independent mean groups for parametric data was done using Student $t$ test while the comparison between 2 dependent groups for parametric data was done using Paired $t$ test. Chi-square test was used to study the association between each 2 variables or comparison between 2 independent groups as regards the categorized data. The probability of error at 0.05 was considered significant $(\mathrm{P}<0.05)$, while at 0.001 was considered highly significant $(\mathrm{P}<0.001)$.

\section{Results}

Forty patients, 8 males and 32 females were included in this study, 16 patients had bilateral CTS. Patients were randomly assigned into one of two treatment groups. The demographic data baseline characteristics of patients in both groups are given in (Table 1), which shows non-significant difference between them.

Table 1: Demographic data and baseline characteristics of the 2 groups.

\begin{tabular}{|l|c|c|c|}
\hline \multicolumn{1}{|c|}{ Variable } & Group A & Group B & P-value \\
\hline Number (patients, hands) & 20,28 & 20,28 & 1 \\
\hline Right: left & $20: 8$ & $20: 8$ & 1 \\
\hline Female: Male & $16: 4$ & $16: 4$ & 1 \\
\hline Grade 2 (mild) CTS: Grade 3 (moderate) CTS & $7: 21$ & $9: 19$ & 0.422 \\
\hline Mean age in years (SD) & $51.1(6.9)$ & $49.3(5.7)$ & 0.526 \\
\hline Mean symptoms duration in months (SD) & $10.1(4.7)$ & $11.7(3.4)$ & 0.844 \\
\hline
\end{tabular}

CTS:carpal tunnel syndrome

At baseline, there were no statistically significant differences $(\mathrm{P}>0.05)$ between the two groups regarding the clinical (pain VAS, SSS \& FSS) and the electro-physiological (median nerve DML \& DSL) parameters (Table 2).

Table 2: Baseline mean scores of pain VAS, hand grip strength, SSS, FSC, DML and DSL of the 2 groups.

\begin{tabular}{|l|c|c|c|}
\hline \multicolumn{1}{|c|}{ Variable } & Group A & Group B & P-value \\
\hline Pain VAS score & $7.3(0.8)$ & $7.0(0.5)$ & 0.722 \\
\hline SSS score & $2.8(0.5)$ & $2.6(0.2)$ & 0.562 \\
\hline FSC score & $2.6(0.3)$ & $2.5(0.4)$ & 0.624 \\
\hline Hand grip strength (kg) & $20.1(2.9)$ & $21.4(3.5)$ & 0.672 \\
\hline Median nerve DML (ms) & $4.9(0.3)$ & $4.7(0.6)$ & 0.583 \\
\hline Median nerve peak DSL (ms) & $4.4(0.4)$ & $4.1(0.9)$ & 0.260 \\
\hline
\end{tabular}

VAS: visual analogue scale, SSS: symptoms severity scale, FSC: functional status scale, DML: distal motor latency, DSL: distal sensory latency.

Evaluation of clinical outcome one month after treatment revealed significant symptomatic and functional improvement as documented by the highly significant reduction in pain VAS, SSS and FSS scores $(\mathrm{P}<0.001)$ and the significant elevation of the hand grip scores $(\mathrm{P}<0.05)$ in both study groups as compared to their baseline values (Tables 3 \& 4). Electro-physiologically, median nerve DML and DSL showed highly statistically significant reduction $(\mathrm{P}<0.001)$ in both groups one month following the treatment as compared to their baseline values (Tables $3 \& 4)$. 
Table 3: Comparison between the mean scores of pain VAS, hand grip strength, SSS, FSC, DML and DSL of Group A before and after treatment.

\begin{tabular}{|l|c|c|c|}
\hline \multicolumn{1}{|c|}{ Variable } & Before & After & P-value \\
\hline Pain VAS score & $7.3(0.8)$ & $4.1(0.7)$ & 0.000 \\
\hline SSS score & $2.8(0.5)$ & $1.7(0.3)$ & 0.000 \\
\hline FSC score & $2.6(0.3)$ & $1.6(0.7)$ & 0.000 \\
\hline Hand grip strength (kg) & $20.1(2.9)$ & $25.9(3.5)$ & 0.005 \\
\hline Median nerve DML (ms) & $4.9(0.3)$ & $4.1(0.6)$ & 0.000 \\
\hline Median nerve peak DSL (ms) & $4.4(0.4)$ & $3.6(0.5)$ & 0.000 \\
\hline
\end{tabular}

VAS: visual analogue scale, SSS: symptoms severity scale, FSC: functional status scale, DML: distal motor latency, DSL: distal sensory latency.

Table 4: Comparison between the mean scores of pain VAS, hand grip strength, SSS, FSC, DML and DSL of Group B before and after treatment.

\begin{tabular}{|l|c|c|c|}
\hline \multicolumn{1}{|c|}{ Variable } & Before & After & P-value \\
\hline Pain VAS score & $7.0(0.5)$ & $3.6(0.9)$ & 0.000 \\
\hline SSS score & $2.6(0.2)$ & $1.6(0.4)$ & 0.000 \\
\hline FSC score & $2.5(0.4)$ & $1.4(0.6)$ & 0.000 \\
\hline Hand grip strength (kg) & $21.4(3.5)$ & $27.6(4.7)$ & 0.006 \\
\hline Median nerve DML (ms) & $4.7(0.6)$ & $3.9(0.4)$ & 0.000 \\
\hline Median nerve DSL (ms) & $4.1(0.9)$ & $3.5(0.2)$ & 0.000 \\
\hline
\end{tabular}

VAS: visual analogue scale, SSS: symptoms severity scale, FSC: functional status scale, DML: distal motor latency, DSL: distal sensory latency.

The differences between the two groups regarding the clinical and electro-physiological parameters one month after treatment were statistically non-significant $(\mathrm{P}>0.05)$ as shown in (Table 5).

Table 5:Comparison between the mean scores of pain VAS, hand grip strength, SSS, FSC, DML and DSL of both groups after treatment.

\begin{tabular}{|l|c|c|c|}
\hline \multicolumn{1}{|c|}{ Variable } & Group A & Group B & P-value \\
\hline Pain VAS score & $4.1(0.7)$ & $3.6(0.9)$ & 0.122 \\
\hline SSS score & $1.7(0.3)$ & $1.6(0.4)$ & 0.455 \\
\hline FSC score & $1.6(0.7)$ & $1.4(0.6)$ & 0.657 \\
\hline Hand grip strength (kg) & $25.9(3.5)$ & $27.6(4.7)$ & 0.426 \\
\hline Median nerve DML (ms) & $4.1(0.6)$ & $3.9(0.4)$ & 0.224 \\
\hline Median nerve DSL (ms) & $3.6(0.5)$ & $3.5(0.2)$ & 0.824 \\
\hline
\end{tabular}

VAS: visual analogue scale, SSS: symptoms severity scale, FSC: functional status scale, DML: distal motor latency, DSL: distal sensory latency.

\section{Discussion}

According to the American Academy of Neurology, CTS is a common disease and there is a 10\% lifetime risk of developing this pathology. At the neutral position of the wrist, carpal tunnel pressure is $3 \mathrm{~mm} \mathrm{Hg}$ in normal while it is $32 \mathrm{~mm} \mathrm{Hg}$ in patients with CTS. The pathophysiology of the nerve lesion is ischemic and is due to compression of the vasa nervorum secondary to the increased pressure [21].

Several investigators have reported the beneficial effect of LSI in the treatment of mild to moderate CTS. It is believed to reduce perineural inflammation or soft tissue swelling and may stabilize the neural membrane, Improvement of symptoms may be seen several days after the injection. In severe cases, steroid injection is insufficient to relieve the pressure on the nerve, it may be used only before surgery, [7,21,22]. However, LSI may lead to serious median nerve injury if not performed correctly.

In our study, the therapeutic effect of Dex-P phonophoresis as a conservative treatment method for mild to moderate CTS was compared to the effect of local Dex-P injection. At one month following local corticosteroid treatment either by phonophoresis or by LSI, our patients in both groups reported significant symptomatic improvement as documented by the decrement in pain VAS and SSS scores and they also reported significant functional improvement as documented by the decrease in FSS score. Our study also showed that there was a significant 
objective improvement in the hand grip strength and in EDX data (DML \& DSL of the median nerve) in both groups.

Spontaneous improvement of neurophysiological measurements in CTS has been demonstrated at 15 months' follow-up [13]. Improvement of EDX results in our study at 1 month after treatment suggests that it is more likely due to the local corticosteroid treatment rather than spontaneous improvement.

Our results were similar to those reported by Karatay and his colleagues [23] who reported statistically significant improvements one month after treatment in both clinical parameters (nocturnal pain \& BCTQ) and EDX data (DSL \& DML) of median nerve in patients treated with either LSI or phonophoresis. After 6 months, the values of all parameters remained significant in LSI group, while significant differences were found only in clinical parameters in phonophoresis group. In this study 45 patients with bilateral mild CTS (90 hands) were randomized into 3 groups each one contained 15 patients (30 hands): LSI, steroid phonophoresis and steroid iontophoresis groups. Patients were evaluated for clinical and EDX parameters at baseline, $1 \& 6$ months after treatment. In LSI group, $4 \mathrm{mg}$ Dex$\mathrm{P}$ without local anaesthetic was injected. In phonophoresis group $0.1 \%$ Dex-P pomade via ultrasound waves, $1 \mathrm{MHz}$ frequency, $1 \mathrm{~W} / \mathrm{cm}^{2}$ intensity, pulsed mode $1: 4$, with a transducer of $5 \mathrm{~cm}^{2}$ was applied for 10 minutes per session, 5 days/week for 3 weeks.

Our results were different from those reported by Aygül and his co-workers [10] who concluded the superior efficacy of LSI compared to steroid phonophoresis in the treatment of CTS. In this study 56 hands of 31 patients with mild to moderate CTS were randomly treated with LSI, phonophoresis or iontophoresis. BCTQ \& EDX for CTS diagnosis were performed on the initial visit and at 2 and 4 months after treatment. Phonophoresis treatment was administered for 10 minutes per session at a frequency of $3 \mathrm{MHz}$ and an intensity of $1.0 \mathrm{~W} / \mathrm{cm}^{2}$, with a transducer of $5 \mathrm{~cm}^{2}$ and with a mixture of $0.1 \%$ Dex-P, 5 days/week for 3 weeks. One $\mathrm{ml}(4 \mathrm{mg})$ Dex-P was used for LSI. At 2 \& 4 months follow up, the was statistically significant differences in both BCTQ \& EDX parameters in LSI group while in phonophoresis group, it was found only in BCTQ parameters. These results were different from those reported by Soyupek et al. [9] who compared the efficacy of NSAID phonophoresis\& steroids phonophoresis in the treatment of CTS to the efficacy of LSI and to that of splinting with a 3-month follow-up. Eighty-four hands of 51 patients with CTS were randomized into 4 treatment groups. Pain VAS, handgrip strength, hand function tests \& EDX were done before and 3 months after treatment. Phonophoresis therapy was performed daily, 5 times a week for 3 weeks for 10 min per session at a frequency of $3 \mathrm{MHz}$ and an intensity of $1.5 \mathrm{~W} / \mathrm{cm}^{2}$, with $5 \mathrm{~cm}^{2}$ transducer using betamethasone valerate $0.1 \%$ ointment, while $0.5 \mathrm{mg}$ of betamethasone dipropionate was used for LSI. Significant improvement was observed in steroid phonophoresis group concerning EDX findings, hand grip strength, and hand function tests but not in pain VAS, while in LSI group significant improvement was observed in pain VAS and hand function tests only.

Bakhtiary and his colleagues [4] conducted a randomized clinical trial on 34 patients with mild to moderate CTS (52 affected hands). Patients were randomly assigned in phonophoresis or iontophoresis groups. Participants in each group received 10 treatment sessions of either iontophoresis or phonophoresis of $0.4 \%$ Dex-P on their wrist region. In the phonophoresis group, aquasonic gel containing $0.4 \%$ Dex-P was applied and pulsed (20\%) ultrasound waves with $5-\mathrm{cm}^{2}$ probe were used with an intensity of $1.0 \mathrm{~W} / \mathrm{cm}^{2}$ at a $1 \mathrm{MHz}$ frequency for 5 minutes to transfer the medication. The therapy was applied for $5 \mathrm{~min} / \mathrm{session}, 5 \mathrm{~d} / \mathrm{week}$, for 2 weeks. Outcome measures were pain VAS, pinch strength, hand grip strength and EDX. All measurements were performed before the $1^{\text {st }}$ treatment session, at the end of therapy, and after 4 weeks' follow-up. They reported statistically significant improvement in all assessment measures in the phonophoresis group more than the iontophoresis group, which was sustained in the follow-up period and they concluded that phonophoresis of Dex-P treatment was more effective than iontophoresis of Dex-P for treatment of mild to moderate CTS. Gurcay et al. [24] also compared phonophoresis and iontophoresis of $0.1 \%$ betamethasone in conjunction with wrist splint with results obtained with wrist splint use alone in 52 female patients (housewives) with mild to moderate CTS who were randomized in the 3 treatment groups. The SSS and hand grip strength test, were applied before and at the 3rd month after treatment. They reported significant reduction in SSS score in the phonophoresis group only, while there was no significant improvement in hand grip strength in all groups. Ultrasound parameters in the phonophoresis group were $1 \mathrm{MHz}$ and an intensity of $1 \mathrm{~W} / \mathrm{cm}^{2}$, in the continuous mode with $5 \mathrm{~cm}^{2}$ transducer, applied for $10 \mathrm{~min} / \mathrm{session}, 3$ days/week, for 3 weeks.

The different results reported on the effect of corticosteroid phonophoresis in the treatment of CTS by the previous studies may be due to different types \& concentrations of corticosteroids used, a wide range of therapeutic 
parameters such as wave length, exposure intensity, session duration, frequency \& total treatment period and different methods of local application used in these studies. There is no standard therapeutic parameters of corticosteroids phonophoresis for the treatment of mild to moderate CTS.

Regarding our phonophoresis parameters, we used Dex-P because it is a strong long acting corticosteroid that can be used without attenuating the ultrasound energy as it is delivered to the tissues [8], we used the 5- $\mathrm{cm}^{2}$ probe in continuous mode with $1 \mathrm{MHz}$ frequency considering the depth of median nerve in the carpal tunnel as the half-value depth (the depth at which $50 \%$ of the original energy remains) of penetration of US in a tissue, perpendicular to it is $9 \mathrm{~mm}$ for $1 \mathrm{MHz}$ and $3 \mathrm{~mm}$ for $3 \mathrm{MHz}$ and the $10 \%$ value depth for the same tissue would be $30 \mathrm{~mm}$ and $10 \mathrm{~mm}$ respectively [25] and in our opinion, the patient could gain more benefit from the heating effect of the continuous mode. Regarding the session duration, frequency \& total treatment period, we used the usual protocol used in our department for therapeutic ultrasound treatment.

Phonophoresis of corticosteroids could elicit anti-inflammatory and tissue-stimulating effects, it may has the potential to accelerate normal resolution of inflammation and it may accelerate the healing process in damaged tissues [4,24]. The advantages of corticosteroids phonophoresis are: being (painless, noninvasive $\&$ sterile), also it provides adequate local and little systemic concentration of corticosteroids, so it avoids its systemic complications, and it is cheap compared with many other forms of treatment for CTS.

The limitations of our study were limited number of patients and short term follow up. Further studies with larger number of patients, extended follow up, additional use of musculoskeletal ultrasound as objective assessment tool and a study design comparingphonophoresis with therapeutic ultrasound alone may permit more definitive conclusions about the relative effectiveness of this treatment modality.

\section{Conclusion}

Phonophoresis of corticosteroids seems to be an effective conservative treatment method for patients with mild to moderate CTS. It is a safer method and has no complications or side effects. For these reasons, it may be an alternative treatment to LSI.

\section{Financial support and sponsorship}

Nil.

\section{Conflict of interest}

There are no conflicts of interest.

\section{References}

1. Ajeena IM, Al-Saad RH, Al-Mudhafar A, Hadi NR, Al-Aridhy SH.Ultrasonic assessment of females with carpal tunnel syndrome proved by nerve conduction study. Neural Plast 2013; 2013: 754564.

2. Ntani G, Palmer KT, Linaker C, Harris EC, Van der Star R, Cooper C, Coggon D.Symptoms, signs and nerve conduction velocities in patients with suspected carpal tunnel syndrome. BMC MusculoskeletDisord. 2013; 14: 242.

3. Zhang W, Johnston JA, Ross MA, Coakley BJ, Gleason EA, Dueck AC, Santello M. Effects of carpal tunnel syndrome on adaptation of multi-digit forces to object mass distribution for whole-hand manipulation. $\mathbf{J}$ NeuroengRehabil 2012; 9: 83.

4. Bakhtiary AH, Fatemi E, Emami M, Malek M.Phonophoresis of dexamethasone sodium phosphate may manage pain and symptoms of patients with carpal tunnel syndrome.Clin J Pain 2013; 29(4): 348-53.

5. Scanlon A, Maffei J. Carpal tunnel syndrome. J NeurosciNurs 2009; 41: 140-7.

6. Wang YJ, Yan SH.Improvement of diagnostic rate of carpal tunnel syndrome with additional median-to-ulnar comparative nerve conduction studies. Acta Neurol Taiwan 2013; 22(4): 152-7.

7. EL-Badawy MA. Electrophysiological and clinical comparison of local steroid injection by means of proximal versus distal approach in patients with mild and moderate carpal tunnel syndrome. Egypt RheumatolRehabil 2015; 42: 120-7

8. Saliba S, Mistry DJ, Perrin DH, Gieck J, Weltman A.Phonophoresis and the absorption of dexamethasone in the presence of an occlusive dressing. J Athl Train. 2007; 42(3): 349-54.

9. Soyupek F, Kutluhan S, Uslusoy G, Igun E, Eris S, Askin A. The efficacy of phonophoresis on electrophysiological studies of the patients with carpal tunnel syndrome. Rheumatol Int. 2012; 32(10): 3235-42. 
10. Aygül R, Ulvi H, Karatay S, Deniz O, Varoglu AO. Determination of sensitive electrophysiologic parameters at follow-up of different steroid treatments of carpal tunnel syndrome. J ClinNeurophysiol 2005; 22(3): 222-30.

11. Bland JD. Do nerve conduction studies predict the outcome of carpal tunnel decompression? Muscle Nerve (2001); 24: 935-40.

12. American Association of Electrodiagnostic Medicine, American Academy of Neurology, American Academy of Physical Medicine and Rehabilitation. Practice parameter for electrodiagnostic studies in carpal tunnel syndrome: summary statement. Muscle Nerve 2002; 25(6): 918-22.

13. Hagebeuk EE, De Weerd AW. Clinical and electrophysiological follow-up after local steroid injection in the carpal tunnel syndrome. ClinNeurophysiol 2004; 115(6): 1464-8.

14. Practice parameter for carpal tunnel syndrome (summary statement). Report of the Quality Standards Subcommittee of the American Academy of Neurology. Neurology 1993; 43(11): 2406-9.

15. Chen SF, Huang CR, Tsai NW, Chang CC, Lu CH, Chuang YC, Chang WN. Ultrasonographic assessment of carpal tunnel syndrome of mild and moderate severity in diabetic patients by using an 8-point measurement of median nerve cross-sectional areas. BMC Med Imaging 2012; 12: 15.

16. Kabiraj MM, Al-Rajeh S, al-Tahan AR, Abdulijabbar M, Al-Bunyan M. Motor terminal latency index in carpal tunnel syndrome. East Mediterr Health J. 1999; 5(2): 262-7.

17. Bland JD. A neurophysiological grading scale for carpal tunnel syndrome. Muscle Nerve. 2000; 23(8): 1280-3.

18. Gallagher EJ, Liebman M, Bijur PE. Prospective validation of clinically important changes in pain severity measured on a visual analog scale. Ann Emerg Med. 2001; 38(6): 633-8.

19. Levine DW, Simmons BP, Koris MJ, Daltroy LH, Hohl GG, Fossel AH, Katz JN. A self-administered questionnaire for the assessment of severity of symptoms and functional status in carpal tunnel syndrome. $\mathrm{J}$ Bone Joint Surg Am. 1993;75(11):1585-92.

20. Meirelles LM, Santos JBG, Santos LL, Branco MA, Faloppa F, Leite VM, Fernandes CH. Evaluation of Boston questionnaire applied at late post-operative period of Carpal Tunnel Syndrome operated with the paineretinaculatome through palmar port. AstaOrtop Bras. 2006; 14: 126-32.

21. Gökoğlu F, Fındıkoğlu G, Yorgancoğlu ZR, Okumuş M, Ceceli E, Kocaoğlu S.Evaluation of iontophoresis and local corticosteroid injection in the treatment of carpal tunnel syndrome.Am J Phys Med Rehabil. 2005; 84(2): 92-6.

22. Visser LH, Ngo Q, Groeneweg SJ, Brekelmans G. Long term effect of local corticosteroid injection for carpal tunnel syndrome: a relation with electrodiagnostic severity. ClinNeurophysiol. 2012; 123(4): 838-41.

23. Karatay S, Aygul R, Melikoglu MA, Yildirim K, Ugur M, Erdal A, Akkus S, Senel K. The comparison of phonophoresis, iontophoresis and local steroid injection in carpal tunnel syndrome treatment. Joint Bone Spine. 2009; 76(6): 719-21.

24. Gurcay E, Unlu E, Gurcay AG, Tuncay R, Cakci A. Assessment of phonophoresis and iontophoresis in the treatment of carpal tunnel syndrome: a randomized controlled trial. Rheumatol Int. 2012; 32(3): 717-22.

25. Cambier D, D'Herde K, Witvrouw E, Beck M, Soenens S, Vanderstraeten G. Therapeutic ultrasound: temperature increase at different depths by different modes in a human cadaver. J Rehabil Med. 2001; 33(5): 212-5. 